\title{
Nasal ganglioglioma
}

INSERM

\section{Source}

INSERM. (1999). Orphanet: an online rare disease and orphan drug data base. Nasal ganglioglioma. ORPHA:141115

Nasal gang liog lioma is a rare tumor, presenting in newborns, containing both neuronal and astrocytic components and that can be endonasal, extranasal or both. It is usually identified as a nasal mass that may cause feeding difficulties and nasal obstruction. 